\title{
ucber $\boldsymbol{f} i \boldsymbol{d}$ t.
}

(Die Biffert begeidnea ble Maragrapben).

\section{Cinleitung.}

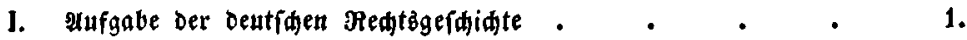

II. Queflen Derfelben . . . . . . . . 2 .

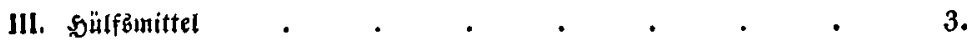

IV. Beffidte der Bearbeitung . . . . . . . 4.

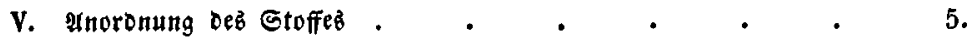

\section{(Erites 3 uch.}

\section{Fedt und :}

I. Die älteftent Beitent.

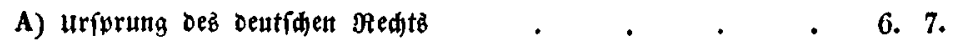

B) Befdaffenfeit Der פation.

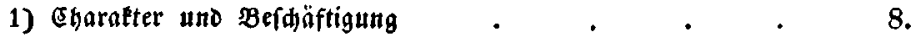

2) Buftand Der Perionen.

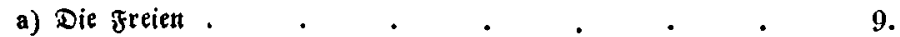

b) Die eoten Befidtedter $\quad . \quad . \quad . \quad . \quad .10 .11$.

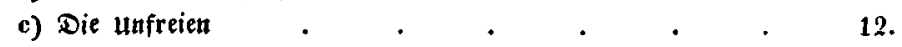

C) Berfaliung.

1) Die Randebgemeinde uno die Sbrigteiten . . . 13. 14.

2) Dab̉ Redht uno der Boleźfriede . . . . 15.16 .

3) Die Fanilie und Die Biutrahe . . . . . 17. 18.

4) Peligion und priefterthun . $\quad . \quad$. $\quad . \quad$. 19.20.

5) Sriegbivefett. Befolgefdaften . . . . 21-23. 
D) Entwiflung ber tönigliden Bewalt $\quad$, . . 24. 25.

11. Berührungen mit ben Römern $\quad . \quad$. 26. 27.

11I. Bilcung oer germanifden Reide im füoliden (Furopa.

A) Daz Seid) oer Burgutoier.

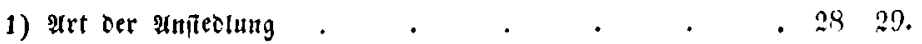

2) Juftano Der Red)tह̉quellett.

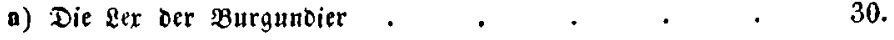

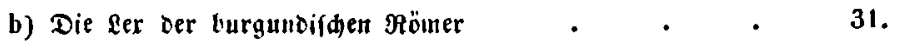

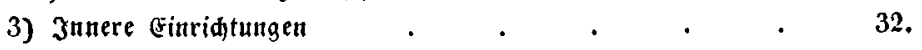

B) Dab Beid) oer neeftgothen.

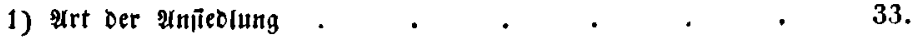

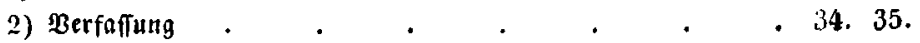

3) 3uftano ber Redtzqueflen.

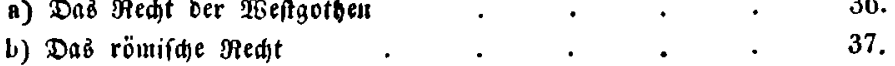

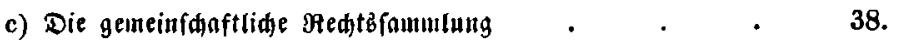

C) 3talien.

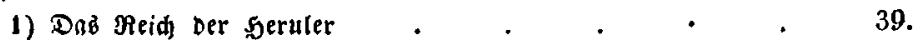

2) Daz Reid oer Ditgothen . . . . . . 40. 41.

3) Die Serrfanft Der Ditrümer $\quad$. $\quad$. $\quad$. 42.

4) Dab Reid Der Langobardent.
a) Irt Der Intifeolung.

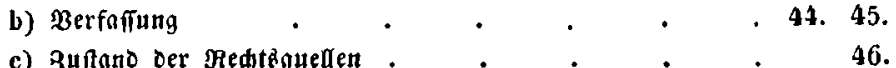

IV. Då̉ fränfifąe Reid.

A) Berdjidte Dez Reidez.
1) Entftebung Deffelben.
2) Theilungen und Erweiterungen
47. 48.
3) Die Erhöfutg Der Farolinger
49. 50.

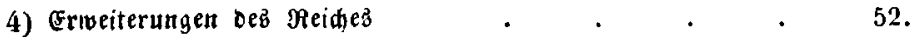
5) Die Serftellung Dez abendändifđen Raiferthumb . . 53.

B) Elemente oer $\mathfrak{B e r f a f f u n g}$.

1) Die Bevölteruttg $\quad . \quad$. $\quad . \quad$. $\quad . \quad 54-56$.

2) Dab Rönigthuut.

a) Beocutung beffelben. Friede. Bant . . . 57-61.

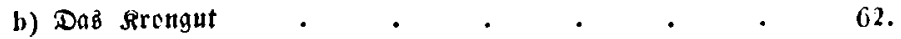

c) Der unterthatentverbano . . . . . . 63.64 .

d) Befolgen. 2utruftionen . $\quad$. $\quad$. $\quad . \quad 65$.

3) Die Rirde. Rlöfter. Rirdengut . . . . . 66. 67.

4) 3uftano Der Perionen.
a) Die Bermanen
$68-70$
b) Die Mümer
71. 72 . 


\section{$\rightarrow \mathrm{XI}-$}

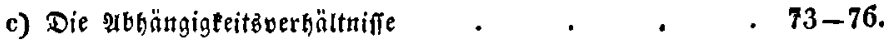

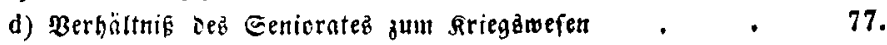

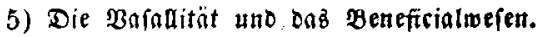
a) Entftehung oer Baiallität
78. 79.
b) Entftehtung Der Beneficien $\quad . \quad$. . . $80-83$.

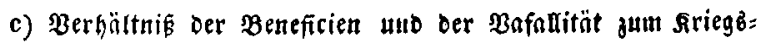

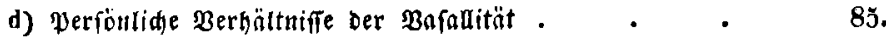
e) Feuere Meinungen über die Bafakität uno die Beneficien 86 .

C) Bon Der Reidbregierung.

1) Drgane für dab ganze Bieid.
a) Der Rï̈tig
87.
b) Urt ber Thronfolge $\quad . \quad$. $\quad . \quad$. $\quad . \quad$. $88-90$.

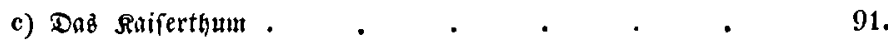
d) Die Reidiz= und 5ofbeanten . . . . . 92-94.
e) Die Reidgtage . . . . . . . $95,96$.

2) Berwaltung Der eingelnen Tfeile.
a) Die fröntifde Bauverfaffuttg
97-105.
b) Die fräntifaen \$erzoge uno Martgrafen
106. 107.
c) Die ३mumunitäten
$108-116$
d) Berbăltnî́ befonterer Bolfäftämme . . . 117-124.
e) Die küniglidien Sendboten $\quad$. . . . . $\quad$. 125 .

3) Bon oen öfentlinen cintinften und enfen.
a) Ertrag Der \&rongüter
126.
b) Steuern
c) Brüdstert. Weggeloer. 3ülle . . . .
127.
d) Miünjwefen
128.
129.
e) Misurnheiftungen
130 .

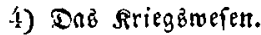
a) Der sjeerbant
$131-135$.
b) Mobilundung Dez Reidabeerez
136.
c) Bertbeidigung der Bränjen und Rirfen. \&anowebr
137.

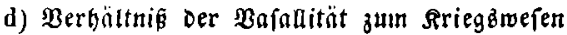
138.
e) Unwanolung oer alten Einridtungen . . , 139.140.

5) Reben uno Gitten.

a) Deffentlide Sidjerheit . $\quad . \quad$. $\quad . \quad$. $\quad 141$.

b) Difciplit ber Sitten . . . . . . . $\quad$. 142.

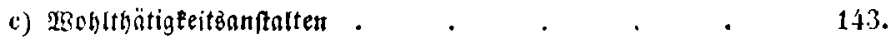

d) Bürgerlider Bertegr . . . . . . . . 14t.

D) 3uthuto oer ßiedtäqueller.

1) Sharafter Deż Redtż . . . . . . 145-149.

2) Eingelne Bullegredte. 


\section{$-\mathrm{XII} \rightarrow$}

a) Die let Balica . . . . . . 150, 151 ,

b) Die ser ber Mituarier . $\quad$. $\quad$. $\quad$, 152.

c) Redtąutellen in fübliqen Gaalien . . . 153.

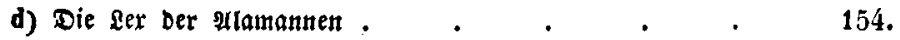

e) Die lex Der Bayern . . . . . . . 155 .

f) Die Let der Anglier, હadien uno friefen . . 156.

g) Redtbąuellen in Der Rombarbei . . . . . 157.

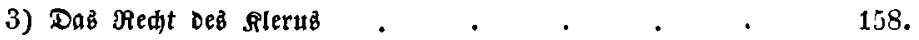

4) Deidägefę̧e uno töniglide Berorontungett . . . 159. 160.

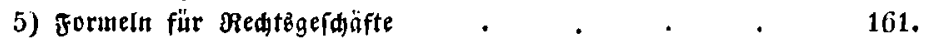

V. Beue 3uftände, welde auf bie unwanthtutg ber Berfaffung cinwirtten.
A) Dic Theilungen bez̉ Reiđez
162. 163.

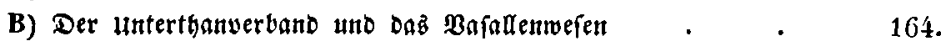

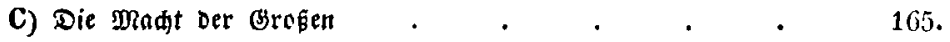

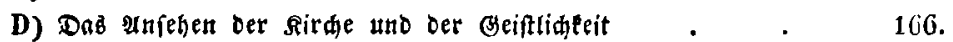

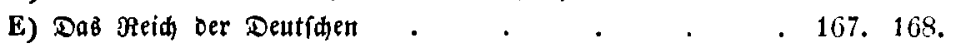

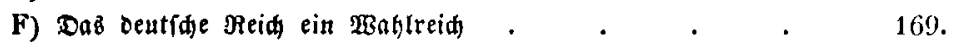

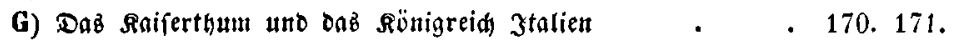

H) 2enberangen it ber provinzinlverfafing.

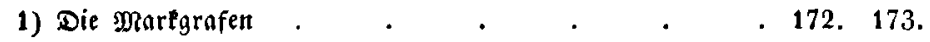

2) Die Serzoge . . . . . . . . . . $174-180$.

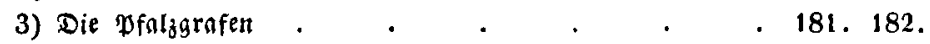

4) Auflöfung Der Baue uno Brafínaften . . . . 183-186.

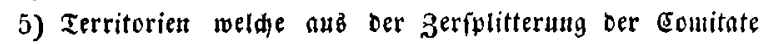
bervorgiengen.

a) Die Gtifte uno avteien.

a) Berbältniffe berfeltuen . . . . . 187-190.

B) Die Gtiftz= 1 tho Rloftervögte . . . . 191-195.

b) Die Braffinften $\quad . \quad$. $\quad . \quad$. $\quad . \quad 196$.

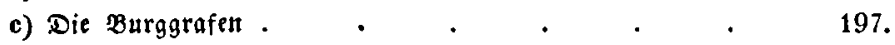

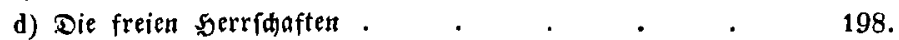

6) Fernere Befdidite Dez Serjogthumb.

a) 2ufröfung ber alten Serzogthïmer . . . 199 .

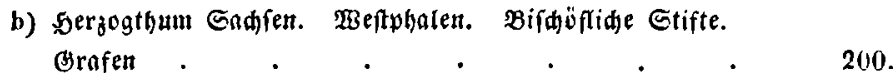

c) Braunfdineig=Rüneburg. Thüringen. Serfen . . 201.

d) Serzogtfum Bayern. Sïrnthen. Steiermar . . 202.

c) Edwaben. 3ähringen. Baden uno Anocre . . 203.

f) Frnnten. NB̈̈rgurg. Notenburg uno 2noere . 204.

g) Oberlotbringet. Brabant. Limburg. Erzftift હöftr.

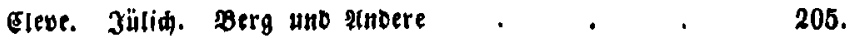


h) Braubenburg. Defterreid $\quad$ - . $\quad$. $\quad 206$.

i) פecflenburg. Poumern. Mügen $\quad$. $\quad$. 207.

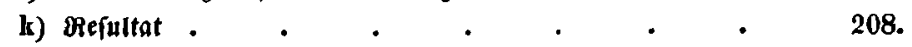

7) Fernere Befdidte ber Pfaljgrafen . . . 209.210.

8) Die Reidgbogteien $\quad . \quad$. . . . . 211.

J) 2renderungen im 3uftande Der Perfonen.

1) Die Pfleghaften uno die Sdjöffenbarfreien . . 212-214.

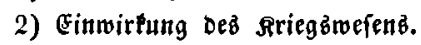

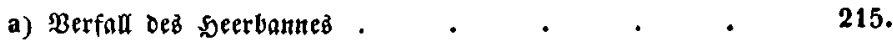

b) 2uftoumen der Feudalmiliz $\quad . \quad$. $\quad . \quad$. 216 .

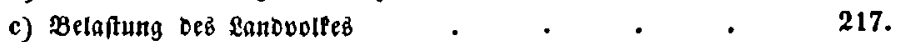

d) Mitter unઠ Bauer $\quad . \quad$. $\quad . \quad$. $\quad$. 218.219.

3) Die Miniterialen.

a) Entftebung dief́e Berbăltnifiez . $\quad . \quad$. 220.221.

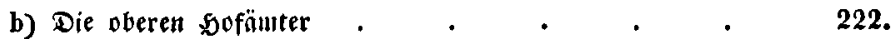

c) Stellung Der MRinifterialen $\quad$. $\quad$. $\quad$. $\quad$. 223.

d) Diente Der Minifterialen $\quad$. $\quad$. $\quad$. 224.

e) Berforgung oer Minifterialen $\quad$. $\quad$. $\quad$. 225.

f) Privatredtliđe Berbältniffe Der Minifterialen . . 226.

g) Itrten oer Minifteriaten . $\quad$. $\quad$. $\quad$ - 227.

h) Untergang der MRinifterialität $\quad . \quad$. $\quad . \quad$. $\quad$ 228. 229.

K) Die Etäote.

1) Die bifqüfliden Stäote . . . . . . . 230-238.

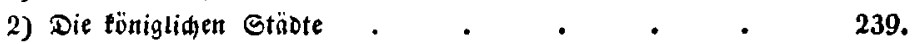

3) Die Territorialfïbte $\quad . \quad$. $\quad . \quad$. 240. 241.

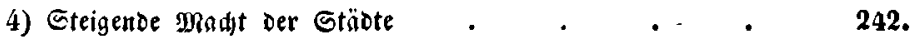

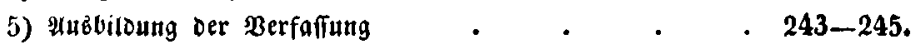

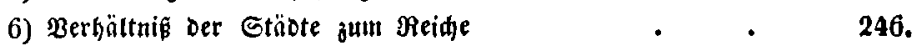

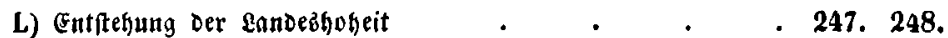

VI. Daz ocutfale Reid in Mittelalter.

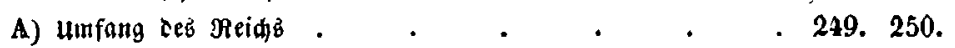

B) Die Reidbregierung.

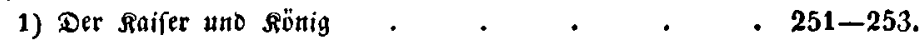

2) Die Reidbz= uno 50 fbeamten . . . . . 254-258.

3) Die Reiđb̀ftünoe.

a) Bezeidnung Derfelben $\quad$. $\quad$. $\quad$. $\quad$ 259-261.

b) Der Rehnảverband der Reiđäftände . . . . 262-265.

4) Die Reidb̈verfanmungen . $\quad . \quad$. $\quad$. $\quad . \quad 266$.

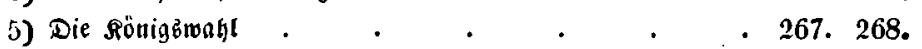

6) Bon den Ginfünften dez ßReidz . . . . 269-272.

7) Bon Dem Reidb̈riegäwefen . . . . 273-276.

8) Reven Hit Sitten . . . . . . 277-279. 


\section{- XIV -}

C) Berwaltung in ben eittzelten Territoriert.

1) Der Randezherr . . . . . 280. 281

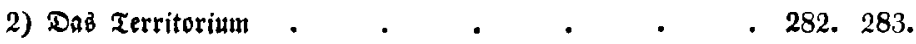

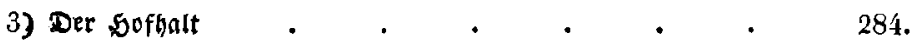

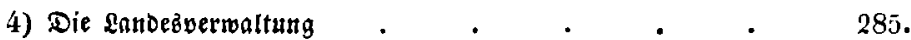

5) Sjoftage. Iandtage. canboing . . . . . 286.287.

6) Berwaltung oer einzelnen Theile.

a) Jär die Erbebung Der Landeaberrliden Einfünfte $\quad$. 288.289.

b) Für Die Redtapflege.

c) Regelmäвiger 3uftand $\quad . \quad$. $\quad$ • . $290,291$.

B) Die geiftliden Territoriett . . . . 292.

y) Wertwgalen und Gadfen $. \quad . \quad . \quad .293 .294$.

c) Befreite mittelbare Befinungen . $\quad$. . . 295. 296.

d) Die Etäbte.$\quad$. $\quad$. $\quad$. $\quad$. $\quad$. 297.

e) Die bäuerlide Berfaffung . . . . . . 298-301.

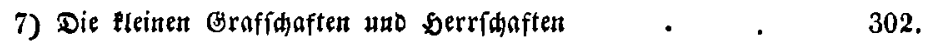

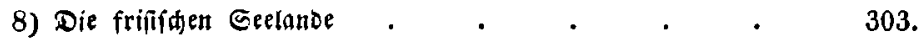

9) Die Lanteäherrliden Einkünfte . . . . 304-307.

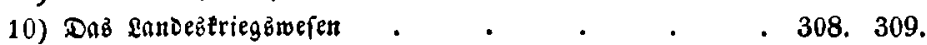

11) Die Eandebpolizei $\quad$. $\quad$. $\quad$. $\quad$. 310.

D) Die Reidjzyogteien unb anderen reidzunmittelbaren (3ebiete 311.

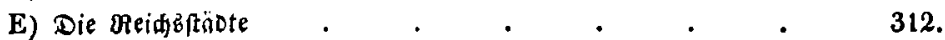

F) 3uftano oer ßiedtżqueller.

1) Beränderungen vom jehnten bis oreizehnten $\mathfrak{J a g h b u n d e r t . ~}$

a) In Deutfhland $\quad . \quad$. $\quad . \quad$. $313-315$.

b) Im Föntrigreid Jtalien . . . . . 316-319.

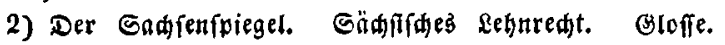

Ridtfteig . . . . . . . . 320-324.

3) Daż Land = und Leguredtabud Doer ber fogenanate

Gajuabenfpiegel . . . . . . 325. 326.

4) $\mathfrak{2}$ ḃ̉biloung ber particularredite.

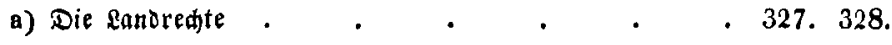

b) Die Etadredte . $\quad . \quad$. $\quad . \quad$. $\quad 329.330$.

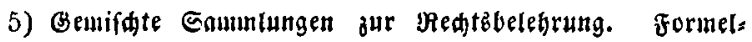

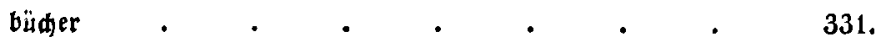

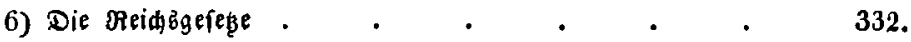

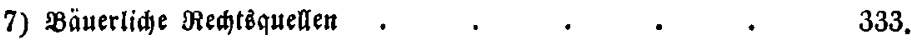

VII. Daz Deutfitye Reid Der neteren 马eit.

A) Neue Finridtungent.

1) 2 bidlula Der Territorialgewalt . . . . . $\quad 334$.

2) Einigungen und $\mathfrak{a n d f r i e d e n t ~ . ~ . ~ . ~ . ~ 3 3 5 . ~} 336$.

3) Erwiger Randriede, oieidğfaumergeridt . . . 337. 
4) Beidbregiment. Sreizeintfeilung . . . . 338.

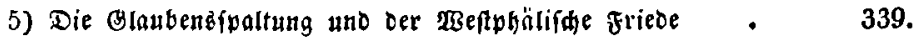

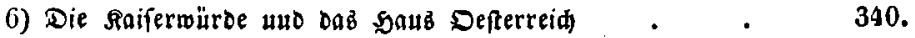

7) Der \&üneviller frieden und feine folgen . . . 341.

B) Die Reidjżregierung.

1) Der Raifer

2) Der Eniferliđe $\mathfrak{S} \mathfrak{c} f$

3) Die Reidebuenuten

4) Dic Rurfürften unt Erzänter . . . . . . 345. 346.

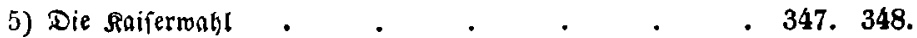

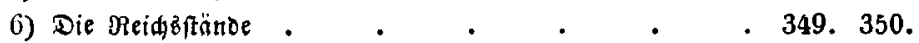

7) Die Reidätage. Reidsbewutationen . . . 351-353.

8) Die Rreigeinridtung . . . . . . 354.

9) Die Reidatriegżverfafing . . . . 355-357.

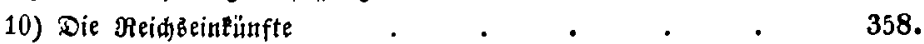

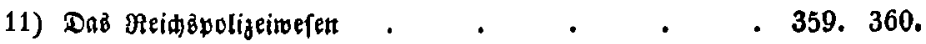

C) Die Territorien.

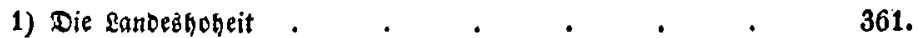

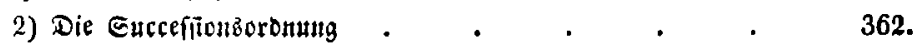

3) Der fürftlide sofbalt . . . . . . . . 363.

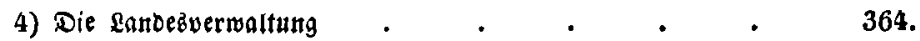

5) Die Ranoftände . $\quad . \quad$. $\quad . \quad \ldots \quad . \quad 365$.

6) Bermaltung Der eimzelnen Iandeatbeile . . . 366. 367.

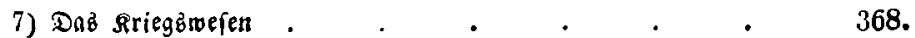

8) Die Iandeăgerrliden Gintlinfte . . . . . 369-373.

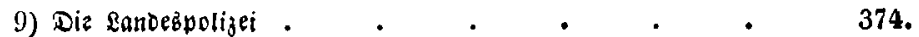

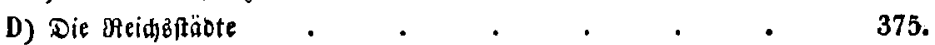

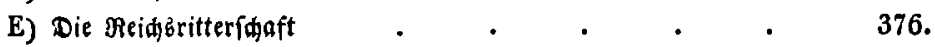

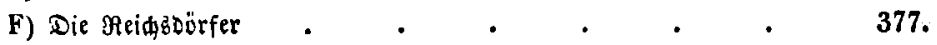

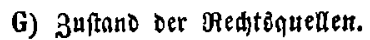

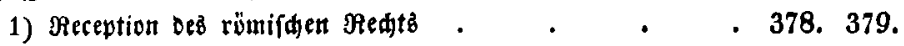

2) Bearbeitung Deż Deutífen Medtż $\quad$ - . . 380 .

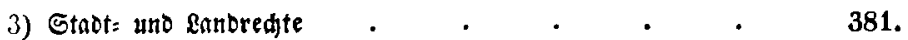

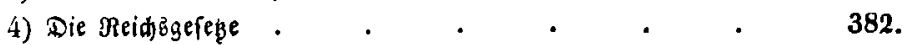

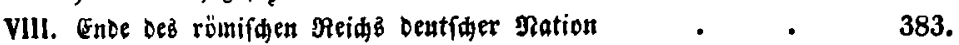

